\title{
A mulher na botânica: questões de gênero na participação feminina em congressos de botânica no Brasil
}

\author{
Natashi Aparecida Lima Pilon ${ }^{1}$ e Giselda Durigan ${ }^{2,3}$
}

Recebido: 5.08.2010; aceito: 17.02.2011

\begin{abstract}
Woman in botany: gender issues in women's participation in botanical congresses in Brazil). Gender and science or gender and knowledge production have been issues of much discussion around the world. In order to examine women's participation in Botanical Science and to verify if some kind of gender bias exists in knowledge production in this area, we analyzed proceedings of three botanical congresses across a period of 21 years. Over this period, unlike other fields of knowledge, an evolution of female participation in research was observed in botany, as well as an increase in knowledge production and changes in the leadership position of women. Botanical Science, therefore, differs in relation to the sciences in general, regarding gender issues.
\end{abstract}

Key words: botanical science, gender proportion, leadership

RESUMO - (A mulher na botânica: questões de gênero na participação feminina em congressos de botânica no Brasil). A questão gênero e ciência, ou gênero e produção de conhecimento, vem sendo alvo de muitas discussões atualmente em todo o mundo. Com o objetivo de analisar a participação feminina na botânica e verificar se há algum tipo de viés de gênero neste campo do conhecimento, foram analisados anais de três congressos de botânica em um intervalo de 21 anos. No período analisado constatou-se a evolução da participação feminina nas pesquisas em Botânica, o aumento da produção de conhecimento e mudanças na posição de liderança das mulheres. A Botânica, portanto, difere em relação às ciências em geral no que diz respeito às questões de gênero.

Palavras-chave: liderança, pesquisas em botânica, proporção de gênero

\section{Introdução}

Questões sobre gênero e ciência constituem um tema amplamente abordado nos últimos anos em todo o mundo. Ainda que as mulheres estejam alcançando proporções inéditas em diferentes áreas do conhecimento, ainda há ampla distância de uma situação de maior equidade em termos de salário e quantidade de profissionais, em comparação com a remuneração e número de homens atuando em cada área (Velho \& León 1998).

Durante toda a história da civilização, as mulheres têm sido minoria no mercado de trabalho, mas discussões de gênero sempre levantam questionamentos relativos à capacidade e à competência. Costa (2006) ilustra esta questão utilizando exemplos como as afirmações de Lawrence Summers, ex-reitor da
Universidade de Harvard, EUA, sobre a incapacidade das mulheres para produzir conhecimento (de serem, portanto, boas cientistas) e a tese de que a inferioridade feminina seria decorrente de diferenças biológicas. Em contrapartida a essas afirmações, Barres (2006), professor de neurobiologia da Universidade de Stanford, atacando a tese de Summers, afirmou que a barreira ao sucesso feminino na ciência deve-se a fatores culturais. Em sua publicação, Barres relatou o fato de ser transexual e que, na adolescência, quando se chamava Bárbara, teria encontrado muitas limitações, sendo, inclusive, desencorajado a buscar vaga no Instituto de Tecnologia de Massachusetts (MIT), onde se formou. Depois que assumiu a identidade masculina, ouviu um pesquisador dizer que "o trabalho de Ben Barres é muito melhor que o de

1. Universidade Estadual Paulista Júlio de Mesquita Filho, Departamento de Ciências Biológicas, Av. Dom Antônio 2100, Caixa Postal 65, 19806-900 Assis, SP, Brasil

2. Instituto Florestal, Floresta Estadual de Assis, Caixa Postal 104, 19802-970 Assis, SP, Brasil

3. Autor para correspondência: giselda@femanet.com.br 
sua irmã" (Revista Fapesp, agosto 2006).

Mesmo depois do surgimento de vários movimentos a partir dos quais ideais feministas foram construídos e uma posição mais significativa da mulher começou a ser defendida, as ciências naturais continuaram sendo um nicho masculino fechado e, freqüentemente, o preço pessoal pago pelas mulheres que almejam esse meio é muito elevado, incluindo, muitas vezes, ter de deixar de lado sua feminilidade ou reprimir seus instintos, para serem aceitas em um mundo masculino (Citele 2000). Tais discrepâncias são ainda mais marcantes nas ciências exatas, nas quais a participação feminina é bem reduzida (Leta 2003). A verdade é que a proporção de mulheres na ciência não reflete a proporção de mulheres economicamente ativas no Brasil - 43\% e 45\%, para os anos de 2003 e 2007 , respectivamente, representando quase a metade da força de trabalho no país (IBGE 2009).

Uma das barreiras que dificultam o sucesso da mulher na carreira científica refere-se ao grau de escolaridade. A oposição histórica encontrada pelas mulheres para obter conhecimento, títulos e postos semelhantes aos dos homens, mesmo possuindo capacidade similar, os mecanismos psicológicos e sociais que mantêm de maneira informal a discriminação, mesmo quando os obstáculos formais são eliminados, mostram os fatores que marcaram a invisibilidade feminina no desenvolvimento da ciência (Citele 2000). Nos últimos 20 anos, as diferenças entre gêneros em termos de acesso à educação diminuíram sensivelmente na maioria dos países da América Latina. Entre os alunos matriculados no ensino superior, a participação dos gêneros é equilibrada ou, até mesmo, favorável às mulheres. Entretanto, maiores níveis de escolaridade não têm garantido oportunidades equivalentes de emprego para mulheres em relação aos homens. À medida que aumenta a hierarquia em termos de progresso nas carreiras científicas, nota-se um decréscimo substâncial do número de mulheres. Dos pesquisadores líderes nas diversas áreas do conhecimento no Brasil, com mais de 65 anos, apenas 33\% são mulheres (Velho \& Prochazka 2003).

Enquanto persistiu a atribuição do caráter iluminista à ciência (neutralidade, racionalidade e objetividade), as mulheres foram excluídas das práticas científicas, pois tais atributos eram considerados como características de natureza masculina. John Lindley, presidente da sociedade Linneana de Londres e professor de Botânica da recém criada Universidade de Londres, em sua aula de inauguração em 1830, afirmou que a Botânica deveria assumir um caráter mais científico e que, para esse fim, a saída das mulheres era imprescidível para o desenvolvimento da ciência (Osada \& Costa 2006). Todavia, aos poucos, a Botânica, em todo o mundo, tornou-se território favorável ao trabalho de mulheres, que no princípio tomavam notas, preparavam ilustrações científicas e trabalhavam na editoração e publicação de resultados de pesquisas, uma vez que os trabalhos de campo, em si, lhes eram vedados (Osada \& Costa 2006). A Botânica era considerada um "objetivo apropriado para mulheres interessadas na natureza desde os anos de 1760 (...), tornando-se um hobby até meados do século XIX, com muitas mulheres colecionando e prensando amostras de samambaias e flores" (Henson 2000). Porém, nessa época, as mulheres eram proibidas de frequentar as universidades. No Brasil, foi em 19 de abril de 1879 que o imperador D. Pedro II abriu as portas da universidade às mulheres, decretando que "é facultada a inscrição aos indivíduos do sexo feminino, para os quais haverá, nas aulas, lugares separados" (Prates 2008).

Em 1808, com a transferência da Coroa portuguesa para o Brasil, que precisava então de desenvolvimento, começou o processo de institucionalização da Ciência, incluindo a Botânica, que aconteceu por iniciativa do Imperador Pedro II, durante o Segundo Reinado (1840-1889) (Nogueira 2000). O século XIX fez do Brasil palco de inúmeras expedições científicas estrangeiras, uma das quais resultou na importante obra denominada Flora Brasiliensis (Nogueira 2000). Os estudos taxonômicos foram baseados em exemplares coletados por 135 colecionadores, dentre os quais havia apenas duas mulheres: Maria Graham e Therese Prinzessin von Bayern (Peixoto \& Filgueiras 2008).

A própria Sociedade Botânica do Brasil - SBB, criada em 1950, com a finalidade de organizar a comunidade atuante neste campo e dar-lhe maior visibilidade política, refletia a desproporção de gêneros vigente na época. Em sua criação, era constituída por 64 homens e apenas seis mulheres (Nogueira 2000), quadro que viria a ser lentamente revertido ao longo do tempo. A eleição da primeira mulher como presidente da SBB (Maria Luíza Porto), em 1982, foi um marco na participação feminina, que passou a dominar nas últimas décadas mesmo os cargos de liderança no campo da Botânica no Brasil. A SBB realiza reuniões anuais, sendo hoje uma instituição de referência para a Botânica brasileira (Nogueira 2000). 
O Congresso Nacional de Botânica, realizado pela SBB anualmente, é o principal forum de participação dos profissionais e estudantes de Botânica no Brasil e, naturalmente, representa o universo de pessoas que atuam neste campo do conhecimento. Por esta razão, os anais desses eventos serviram de base para o presente estudo, que teve como objetivo verificar se existem desequilíbrios entre a proporção de homens e mulheres no campo da Botânica no Brasil, e se eventuais desequilíbrios têm se mantido ao longo do tempo.

\section{Material e métodos}

Foi utilizado como universo amostral o conjunto de resumos apresentados em três Congressos Nacionais de Botânica, realizados pela Sociedade Botânica do Brasil dentro de um intervalo de 21 anos - 1988, 1999, 2009. Para cada evento analisado, os autores dos resumos publicados foram agrupados por gênero, nas seguintes categorias:

a) autores em geral: para verificar se a proporção de homens e mulheres segue a proporção da população em geral ou da proporção de pesquisadores do Brasil (com base nos grupos de pesquisa do Conselho Nacional de Desenvolvimento Científico e Tecnológico - CNPq);

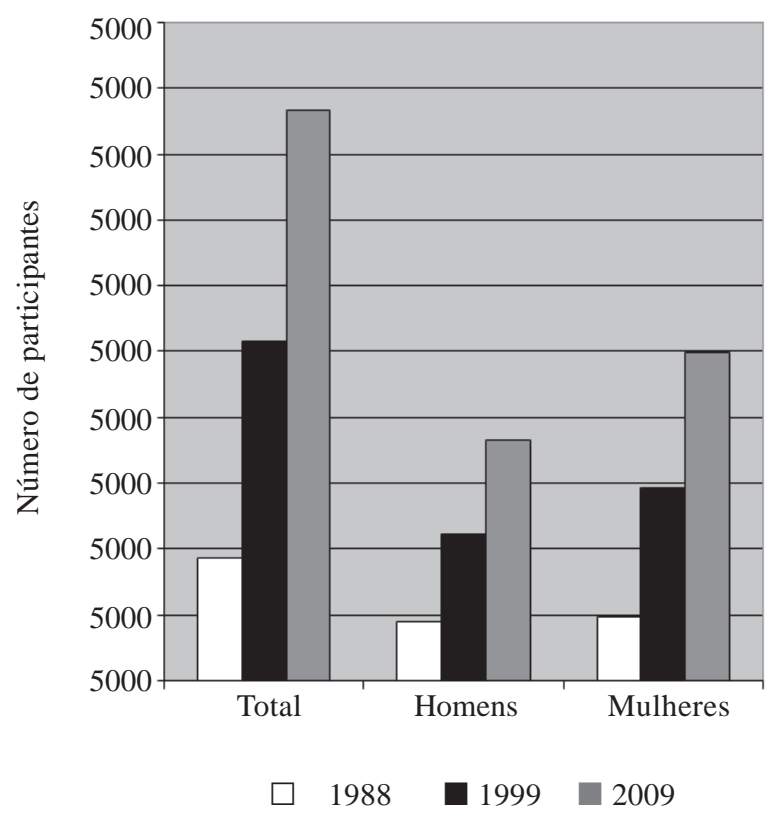

Figura 1. Evolução do número de participantes com resumos publicados em Congressos de Botânica da Sociedade de Botânica do Brasil (1988, 1999, 2009), no total e por gênero.

Figure 1. Number of attendants with abstracts published in Congresses of the Botanical Society of Brazil (1988, 1999, 2009), in total and by gender. b) primeiro autor: para verificar se a liderança nos trabalhos repete aquela dos autores como um todo ou se há viés de gênero;

c) autor individual: para verificar se homens ou mulheres têm maior tendência de pesquisar individualmente.

\section{Resultados e Discussão}

Foram analisados 2.580 resumos, sendo 399 apresentados no Congresso de 1988, 971 no Congresso de 1999, e 1.210 no Congresso de 2009.

Analisando-se o número de homens e mulheres entre os autores em geral (figura 1), verifica-se que houve um notável aumento no número de participantes no evento nessas duas décadas, para ambos os gêneros, refletindo o crescimento da Botânica como ciência no Brasil. Todavia, verifica-se que o número de mulheres entre os autores cresceu mais do que o número de homens, desde 1988.

A proporção de mulheres entre os autores em geral (figura 2) é maior que a de homens e esta diferença tem se acentuado ao longo desses 21 anos. Aumentou de 51,6\% em 1988 para 56,8\% em 1999 e 57,7\% em 2009. Este resultado pode estar relacionado com a suposta aptidão das mulheres para o trabalho botânico,

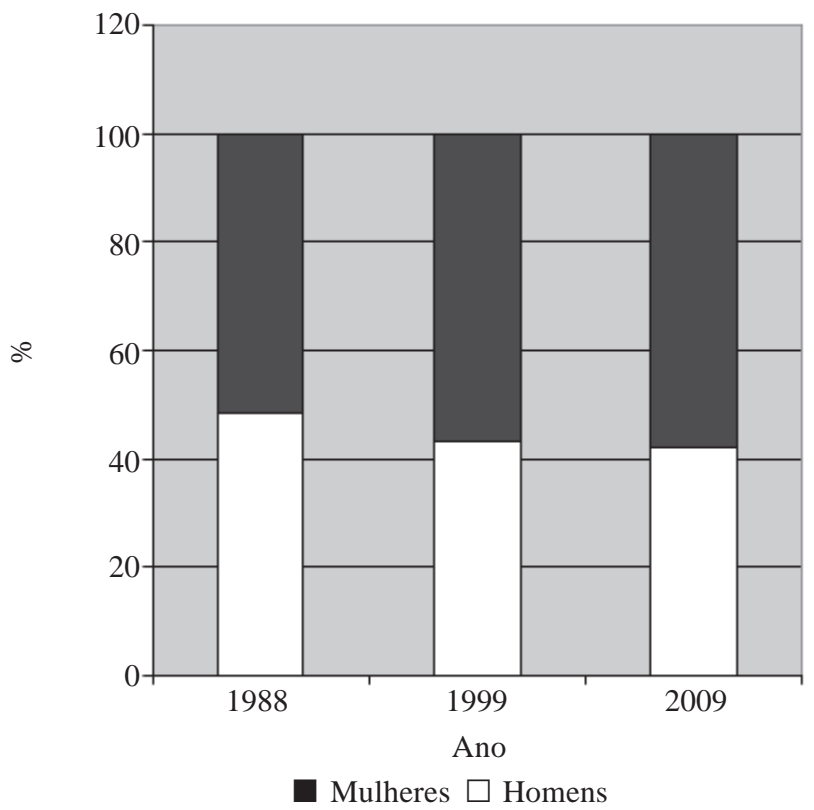

Figura 2. Proporção de homens e mulheres entre os autores de resumos publicados nos Anais dos Congressos de Botânica da Sociedade Botânica do Brasil (1988, 1999, 2009).

Figure 2. Percentage of men and women among the authors of abstracts published in the Annals of the Congresses of the Botanical Society of Brazil (1988, 1999 and 2009). 
como observado por Henson (2000), ao mencionar que a atividade era apropriada para as mulheres, que tinham como passatempo colecionar e prensar samambaias e flores silvestres desde o século XVIII.

Em entrevista concedida a Azevedo et al. (2008), a botânica Leda Dau, considera que a maior atuação feminina na Botânica poderia estar ligada com a delicadeza, o fato de as mulheres serem atraídas pelas plantas, seduzidas pelo aroma. Resposta semelhante também foi obtida no estudo de Barbosa (2006), em que outra entrevistada afirma que a Botânica é uma profissão delicada, característica culturalmente atribuída às mulheres. $\mathrm{O}$ que se verifica é que as próprias mulheres consideravam a Botânica uma profissão que seria apropriada para mulheres.

Talvez nos séculos passados os trabalhos de campo, que de forma rotineira os botânicos sempre realizaram, desde as expedições dos grandes naturalistas, fossem realizados por equipes de apoio e as mulheres botânicas se limitassem a estudar as plantas secas. De outra forma, não há como aceitar a explicação de que tais trabalhos constituam uma prática "delicada", uma vez que com relativa freqüência os botânicos trabalham sob chuva, realizam longas caminhadas transportando pesados equipamentos, coletando material de árvores de grande porte, epífitas, palmeiras e outros grupos especiais de plantas e ainda transportam o próprio material coletado. Ou seja, as atividades de um botânico não têm nada do que se rotula de feminino ou delicado. Na verdade, o que é evidenciado é uma ideologia social atribuindo a esta profissão um caráter feminino, talvez por exigir habilidades associadas ao sexo feminino, como a paciência e a sensibilidade para reconhecer detalhes anatômicos e morfológicos das plantas.

Sendo a Botânica um ramo das ciências biológicas, cabe a observação de Schiebinger (2008) de que, depois da medicina, a biologia foi o campo que apresentou mais alterações no número de homens e mulheres para a análise de gênero ao longo do tempo. Segundo a autora, tais transformações podem ter sido provocadas por uma crescente consciência de que a superação do preconceito de gênero poderia melhorar a ciência. Uma vez que a biologia lida com sexo e gênero, e também porque a biologia foi aberta às mulheres (atualmente $45 \%$ dos doutores são mulheres), os biológos agiram rapidamente para suprimir o evidente preconceito cultural. Porém, ainda que em disciplinas como biomedicina e biologia, que contam com um bom número de mulheres profissionais, tenha havido mais progresso nas questões de produção de conhecimento, esta pode ser uma conclusão precipitada e otimista (Schiebinger 2008).

A razão mais provável para a predominância de mulheres na Botânica no Brasil, porém, pode não estar em habilidades diferenciadas nem em mobilização social pela igualdade de gêneros, mas sim na baixa valorização da profissão. Barbosa (2006), em estudo resultante do projeto Gênero e Ciência: carreira e profissionalização no Instituto Oswaldo Cruz, Museu Nacional e Instituto de Biofísica (1939-1968), apresenta depoimento de algumas entrevistadas que mencionam a predominância de mulheres na Botânica, considerando-a como algo negativo. Afirmam que esse fato seria decorrente da remuneração pouco promissora e, devido a isto, os homens, com o encargo de provedores do lar, escolheriam outras profissões. As mulheres, por não terem essa exigência, procurariam a Botânica, como se fosse um nicho vago.

Dados comprovam a predominância das mulheres nas áreas de ciências biológicas como um todo (tabela 2), mostrando que é superior o número de mulheres em relação ao de homens desde o ingresso nos cursos de Biologia e esta proporção aumenta ainda mais no decorrer do curso, mostrando que a evasão é maior entre os homens. Curiosamente, a proporção de mulheres que concluem o curso de Biologia é ainda maior do que a proporção de mulheres entre os autores nos congressos de botânica, o que elimina a hipótese de que a menor proporção de homens entre os botânicos seja um preconceito masculino em relação à Botânica em si. Caso exista, o preconceito seria em relação à profissão de Biólogo e a predominância de mulheres na Botânica seria apenas uma consequência desse fato.

$\mathrm{Na}$ comparação das figuras 2 e 3 com os censos da comunidade científica em geral, efetuado pelo CNPq (tabela 1), observa-se que a Botânica não segue os padrões de outras áreas científicas nem no universo de profissionais e nem na liderança de pesquisa. Além dos homens serem maioria nas Ciências em geral, a predominância masculina é ainda maior entre os líderes de grupos de pesquisa. O censo do CNPq de 2008, publicado na revista FAPESP (setembro de 2009), demonstra que quando a liderança dos grupos é analisada, a participação feminina cai de $49 \%$ para $45 \%$. Se a comparação for feita por não liderança o percentual de mulheres supera o de homens. No campo da Botânica, a liderança de pesquisa, expressa neste estudo pela proporção como 
Tabela 1. Distribuição de pesquisadores de todas as áreas da ciência por gênero, no Brasil. Fonte: Diretório de Grupos de Pesquisas do CNPq, censos de 1997, 2000 e 2002.

Table 1. Distribution of researchers in all fields science by gender in Brazil. Source: Directory CNPq Research Groups, Census 1997 , 2000 and 2002.

\begin{tabular}{lccccccc}
\hline & \multicolumn{3}{c}{ Pesquisadores } & & \multicolumn{3}{c}{ Pesquisadores líderes } \\
\cline { 2 - 4 } Ano & Total & $\begin{array}{c}\text { Masculino } \\
(\%)\end{array}$ & $\begin{array}{c}\text { Feminino } \\
(\%)\end{array}$ & & Total & $\begin{array}{c}\text { Masculino } \\
(\%)\end{array}$ & $\begin{array}{c}\text { Feminino } \\
(\%)\end{array}$ \\
\hline 1997 & 33.675 & 58,0 & 42,0 & & 10474 & 62,7 & 37,3 \\
2000 & 48.781 & 56,0 & 43,7 & & 16456 & 60,6 & 39,4 \\
2002 & 56.891 & 54,3 & 45,7 & & 21062 & 59,3 & 40,7 \\
\hline
\end{tabular}

Tabela 2. Evolução do número de alunos matriculados e concluintes no ensino superior para o curso de Biologia no período $2000-2003$. Fonte: Inep/ Mec, Instituto Nacional de Estudos e Pesquisas Educacionais Anísio Teixeira.

Table 2. Number of students enrolled in higher education and graduating to the course of Biology in the period 2000-2003.Source: INEP/MEC, National Institute of Educational Studies Anísio Teixeira.

\begin{tabular}{|c|c|c|c|c|c|c|}
\hline \multirow[b]{2}{*}{ Ano } & \multicolumn{3}{|c|}{ Matriculados } & \multicolumn{3}{|c|}{ Concluintes } \\
\hline & Total & Masculino & Feminino & Total & Masculino & Feminino \\
\hline 2.000 & 4.530 & 1.589 & 2.941 & 507 & 136 & 371 \\
\hline 2.001 & 2.686 & 827 & 1.859 & 396 & 100 & 296 \\
\hline 2.002 & 2.736 & 827 & 1.909 & 236 & 55 & 181 \\
\hline 2.003 & 3.807 & 1.173 & 2.634 & 333 & 83 & 250 \\
\hline Total & 13.759 & 4.416 & 9.343 & 1.472 & 374 & 1.098 \\
\hline$\%$ & 100 & 32,1 & 67,9 & 100 & 25,4 & 74,6 \\
\hline
\end{tabular}

primeiro autor dos trabalhos apresentados (figura 3), também é feminina. A proporção de mulheres líderes praticamente manteve-se estável em torno de $60 \%$ ao longo dessas duas últimas décadas, sendo ligeiramente superior à proporção entre gêneros, se analisados os autores como um todo.

O resultado mais surpreendente deste estudo - e de difícil compreensão - foi obtido quando da análise da proporção de homens e mulheres que publicam seus estudos como único autor (figura 4). Entre os botânicos que preferem publicar os resultados de suas pesquisas individualmente, verificou-se forte tendência de aumento, ao longo dos últimos 21 anos, na proporção de autores do sexo masculino. Enquanto a proporção de homens entre os autores em geral foi de $40 \%$ em 2009, a proporção de autores solitários do sexo masculino foi de quase $60 \%$. Ou seja, a proporção de autores solitários do sexo masculino, que não se diferenciava da proporção de homens entre os autores em 1988, sofreu um aumento de cerca de $50 \%$ em duas décadas. A constatação de Teixeira \& Costa (2006), de que homens preferem publicar individualmente, parece se confirmar para a Botânica, tornando-se mais evidente ao longo do tempo. Este resultado merece análises mais profundas para que venha a ser compreendido.

A análise das proporções de gêneros na autoria de trabalhos apresentados nos Congressos Nacionais de Botânica da Sociedade Botânica do Brasil demonstrou que, mesmo sendo a Ciência em geral uma atividade predominantemente masculina, na Botânica as mulheres vêm dominando e aumentando sua produção progressivamente. Resta a dúvida: por que neste aspecto a Botânica apresenta-se como exceção entre as ciências? Será que o tão comentado jeito diferente de fazer ciência, uma ciência feminina, já existe na Botânica? Fica também sem resposta a pergunta: por que está aumentando a proporção de homens que não compartilham a autoria de seus estudos?

Os resultados mostram que há um viés de gênero no campo da Botânica, que reflete o viés que caracteriza as Ciências Biológicas como um todo e que contradiz o observado para o universo dos cientistas no Brasil e no mundo, em que predominam os homens. Há algo a ser compreendido na evolução das relações de gênero na Botânica, que pode apontar 


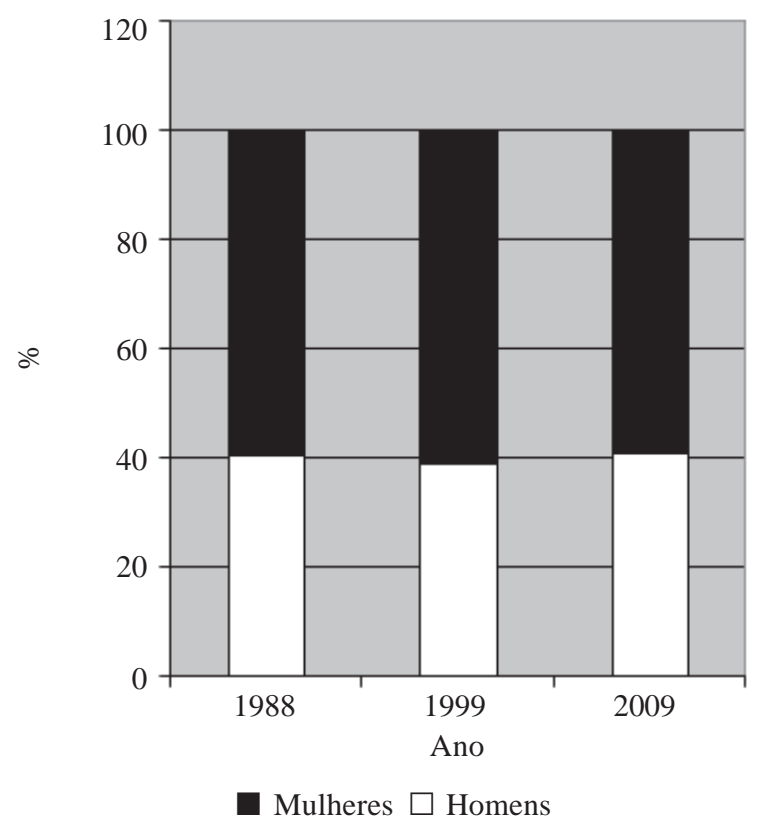

Figura 3. Proporção de homens e mulheres como primeiro autor de resumos publicados nos Congressos de Botânica da Sociedade Botânica do Brasil (1988, 1999, 2009).

Figure 3. Percentage of men and women as first authors of abstracts published in the Congresses of the Botanical Society of Brazil (1988, 1999 and 2009).

caminhos para a busca de igualdade de gênero em outras áreas de conhecimento, onde há, geralmente, exclusão feminina. $\mathrm{Ou}$, talvez, assim como ocorre com as próprias plantas, haja alguma adaptação - ainda não compreendida, a este ou àquele nicho profissional, levando a uma maior concentração de homens e mulheres em determinados campos da ciência e, neste caso, a desproporção entre os gêneros para tais áreas do conhecimento deveria ser aceita com naturalidade.

\section{Agradecimentos}

À professora Maria Tereza Papa Nabão, pelas contribuições durante a construção do trabalho, ao Conselho Nacional de Desenvolvimento Científico e Tecnológico, que, ao instituir o Prêmio Construindo a Igualdade de Gêneros, motivou o desenvolvimento deste estudo e, também, por conceder bolsa de iniciação científica à primeira autora e de produtividade em pesquisa à segunda autora. A dois revisores anônimos e ao Editor, Fábio de Barros, pelas contribuições para melhora do manuscrito.

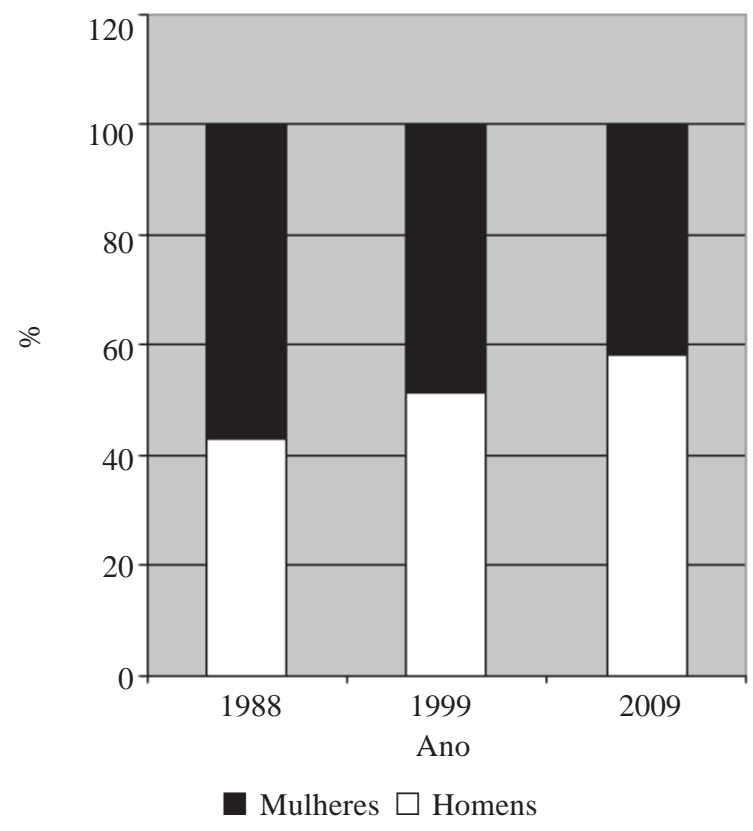

Figura 4. Proporção de homens e mulheres entre os autores que publicaram individualmente nos Congressos de Botânica da Sociedade Botânica do Brasil (1988, 1999, 2009).

Figure 4. Percentage of men and women among the authors who have published individually in the Congresses of the Botanical Society of Brazil (1988, 1999 and 2009).

\section{Literatura citada}

Azevedo, N., Cortes, B.A. \& Sá, M.R. 2008. Um caminho para a ciência: a trajetória da botânica Leda Dau. História, Ciência, Saúde - Manguinhos, Rio de Janeiro 15 (suplemento): 209-229.

Barbosa, S.M. 2006. Gênero e Ciência: um estudo comparativo de seis trajetórias de vida. In: XII Encontro Regional de História- RJ (ANPUH). Usos do Passado: Programa e resumos do XII Encontro Regional de História- RJ. Arquivo Público do Estado do Rio de Janeiro. Niterói, pp. 164-164.

Barres, B.A. 2006. Does gender matter? Nature 442: 133-136.

Citelli, M.T. 2000. Mulheres na Ciência: mapeando campos de estudo. Cadernos Pagu 15: 39-75.

Costa, M.C. 2006. Ainda somos poucas: Exclusão e invisibilidade na ciência. Cadernos Pagu 27: 455-459.

Henson, P. 2000. A invasão da Arcádia: As cientistas no campo da América Latina, 1900-1950. Cadernos Pagu 15: 165-197.

IBGE. 2009. Estatísticas em Educação e trabalho: senso de 2006-2007. Instituto Brasileiro de Geografia e Estatística. http://www.ibge.gov.br (acesso em 09.2009).

INEP/MEC. 2009. Investimentos em C\&T. Instituto Nacional de Estudos e Pesquisas Educacionais Anísio Teixeira. http://www.cnpq.br/servicos/estatisticas/ index.htm (acesso em 09.2009). 
Leta, J. 2003. As mulheres na ciência brasileira: crescimento, contrastes e um perfil de sucesso. Estudos Avançados 17:271-284.

Nogueira, E. 2000. Uma história brasileira da Botânica. Paralelo 15, Brasília; Marco Zero, São Paulo.

Pesquisa Fapesp. 2006. O avanço dos Grupos de Pesquisa. Pesquisa Fapesp 126: 30.

Osada, N.M. \& Costa, M. C. 2006. A construção social do gênero na Biologia. Cadernos Pagu 27: 279-299.

Peixoto, L.P. \& Filgueiras, T.S. 2008. Maria Graham: anotações sobre a flora do Brasil. Acta Botanica Brasilica 22: 992-998.

Prates, P.R. 2008. Mulheres médicas. Revista da Sociedade de Cardiologia do Rio Grande do Sul, 15: 1-4.
Schiebinger, L. 2008. Mais mulheres na ciência: questões de conhecimento. História, Ciência, Saúde Manguinhos 15: 269-281.

Teixeira, R.R.T. \& Costa, P. Z. 2006. Impressões de estudantes universitários sobre a presença das mulheres na ciência. São Paulo.http://www.portal.fae.ufmg.br/seer/ index.php/ensaio/article/ (acesso em 20.10.2009).

Velho, L. \& León, E. 1998. A construção social da produção científica por mulheres. Cadernos Pagu 15: 309-344.

Velho, L. \& Prochazka, M.V. No que o mundo da ciência diferere dos outros mundos? Com Ciência. Revista eletrônica de Jornalismo Científico 50. http:// www.comciencia.br (acesso em 28.09.2009). 